Research Article

\title{
Investigation of Dynamic Load in Superdeep Mine Hoisting Systems Induced by Drum Winding
}

\author{
Jiannan Yao $\mathbb{D}^{1},{ }^{1}$ Xiaojie Deng $\mathbb{D}^{1},{ }^{1}$ Chi Ma $\mathbb{D}^{2},{ }^{2}$ and Tong Xu $\mathbb{D}^{1}$ \\ ${ }^{1}$ School of Mechanical Engineering, Nantong University, Nantong 226019, China \\ ${ }^{2}$ School of Mechanical and Electrical Engineering, China University of Mining and Technology, Xuzhou 221116, China \\ Correspondence should be addressed to Jiannan Yao; yaojiannan1988@163.com
}

Received 3 August 2021; Accepted 25 October 2021; Published 8 November 2021

Academic Editor: Traian Mazilu

Copyright (c) 2021 Jiannan Yao et al. This is an open access article distributed under the Creative Commons Attribution License, which permits unrestricted use, distribution, and reproduction in any medium, provided the original work is properly cited.

Blair mine hoists powered by drum winding are a key equipment in the transportation of a superdeep mine shaft. The step changes of hoisting velocity and acceleration caused by coil and layer crossover of rope winding on the LeBus drum, which will excite impact responses of hoisting systems, cannot be ignored in the hoisting system with high velocity. The paper focuses on investigating the effect of drum winding on impact responses of superdeep mine hoisting systems. Firstly, the hoisting velocity and acceleration were precisely modelled and calculated according to the structure of rope groove of the LeBus drum; secondly, impact responses of the dynamic displacement and load were obtained by applying the established model; eventually, an experiment was performed, and then, the validity of the established model and the response mechanism was verified. The paper will provide good technical support for the design and optimization of the mine hoists in superdeep mine shafts.

\section{Introduction}

Mine hoists are the key equipment in the transportation of mine shafts. With the increase of the mining depth, a Blair mine hoist as shown in Figure 1 will be a good machine to be applied. Higher hoisting velocity and longer length of the hoisting rope are being required in deep mine shafts compared to those in shallow shafts in coal mines. Due to the high velocity and long length of ropes, fluctuations of dynamic load will be obvious in superdeep mine hoisting systems.

The Blair mine hoist in Figure 1 is always powered by LeBus drums, which have parallel grooves with two symmetrical helical crossovers. During the hoisting process, the rope will wind on the drum by coil crossover and layer crossover. The winding radius in the period of coil crossover and layer crossover will change, which will result in the variation of the winded length of the rope and then change the hoisting velocity and acceleration. Due to the high rotating angular velocity, the transition time during the coil crossover and layer crossover will be very small. Furthermore, the increments of the winded length of the rope during the coil crossover and layer crossover are also small and constant, but the transition time during the periods will decrease with the increase of the rotating angular velocity, which will excite step changes of the hoisting velocity and acceleration and even excite load fluctuations. Therefore, the present paper focuses on the investigation of dynamic load in the Blair mine hoist induced by drum winding.

In the researches of the dynamics of the mine hoists, some researchers have done some significant work in recent years. Peng et al. [1] have used the methods of differential geometry, mechanics, and mathematical analysis to study the movements of the crossover and derived the important formula that can reduce the vibration of cable on the drum during the course of crossover. Additionally, they have also studied the transverse vibration characteristics of catenary ropes induced by crossover layouts of multilayer winding grooves in deep mine hoists [2]. However, the variations of hoisting velocity, acceleration, and longitudinal vibration of the rope have not been studied. Yao et al. [3] have derived the functions of the spatial transverse excitation displacements at the drum end; based on this, prediction and reduction of spatial transverse vibration of hoisting catenaries 


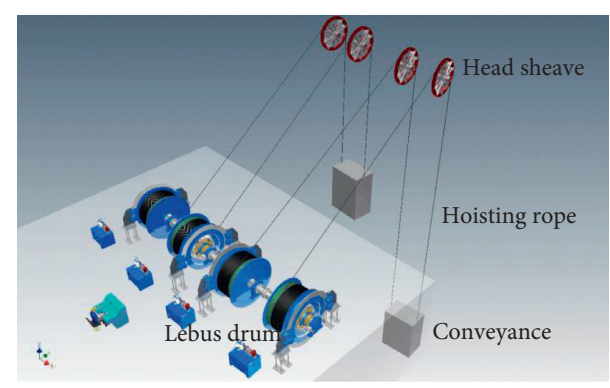

FIGURE 1: Schematic of a Blair mine hoist in superdeep mine hoists.

induced by drum winding in superdeep mine hoists were investigated; however, this work has not discussed the longitudinal impact load of the hoisting rope excited by the drum winding. Wang et al. [4] have analyzed the dynamic behavior of the multicable double drum winding hoister with unbalance factors, the conveyance eccentricity, and the drum radius inconformity by simulation. However, the dynamic behavior of the hoisting rope caused by coil crossover and layer crossover has not been considered. Kaczmarczyk and Ostachowicz $[5,6]$ have employed the classical moving coordinate frame approach and Hamilton's principle to derive a distributed-parameter mathematical model in order to investigate the dynamic behavior of deep mine hoisting cables; nevertheless, the complex motion law of the LeBus drum has not been precisely modelled and has not been considered into the analyses of the dynamic behavior of cables. Wang et al. [7] have investigated the longitudinal response of parallel hoisting systems with timevarying rope length without considering the drum winding and concluded that the system acceleration will cause a relatively large longitudinal vibration in the ropes. Guo et al. [8] have investigated the longitudinal and transverse dynamic responses of a wire rope under periodic excitation in a friction mine hoisting system by performing experiments with different periodic excitation frequencies; however, the results showed that the effect of excitation on the rope tension was not obvious. He [9] has also studied the longitudinal rope dynamics in a friction hoisting system and its coupling effect with friction transmission and concluded that the tension fluctuates obviously, and the dynamic tension regime expands with the friction coefficient increase. Huang et al. [10] have simplified the longitudinal vibration model of a mine hoisting as a second-order underdamped system and obtained its dynamic response in the slipping and unslippage state for a safe emergency barking operation. $\mathrm{Wu}$ and Kou [11] have established the longitudinal-transverse model of mine friction hoist by using of Hamilton's principle, and the modified Galerkin's method has been used to discretize partial differential Equations.

In the other forms of hoisting systems, Cao et al. [12] have studied the coupled vibrations of rope-guided hoisting systems with tension difference between two guiding ropes. Peng et al. [13] have analyzed the transverse and longitudinal vibration characteristics as well as the time-frequency characteristics of the platform frame, car frame, and hoist rope during upward and downward movement through an elevator experimental platform. Zhang et al. $[14,15]$ have built the nonlinear time-varying model of the longitudinal vibration of the hoisting rope using the Hamilton principle and energy method and have studied the effect of lifting quality, traction force, compensating ropes, and tension device on the elevator vibration. Wang et al. [16] have investigated the longitudinal and transverse vibration frequency characteristics of a flexible guiding system under light load, heavy load, and disturbance by generalized- $\alpha$ algorithm and ADAMS simulation. Yang et al. [17] have concerned with the theoretical modeling and experimental verification of the coupled vibrations of building and elevator ropes. Arrasate et al. [18] have investigated the dynamic responses of an elevator system by modeling, simulation, and experimental testing. Zhu and Ren $[19,20]$ have proposed an accurate spatial discretization and substructure method with application to moving elevator cablecar systems.

From the literature studies mentioned above, the longitudinal vibration responses of mine hoisting systems have been concerned by some scholars in considering different influence factors, but few literatures have focused on the load response induced by the motion of the rope on the LeBus drum that is usually applied in superdeep mine hoisting systems. Therefore, a further research should be focused on the impact responses in superdeep mine hoisting systems induced by drum winding.

\section{Description of Excitations from Drum Winding}

LeBus drums with parallel grooves with two symmetrical helical crossovers are usually employed in superdeep mine hoisting systems, and the plane development of the rope winding on the drum is shown in Figure 2. The symmetrical helical crossover grooves make the rope wind on the drum in correct regulation overcoming the shortages of the traditional drums with cylindrical helical grooves such as the rope-jumping and disordered rope arrangement. However, in the hoisting process powered by LeBus drums, the hoisting conveyance is usually lifted by the rope winding on the drum in the patterns of coil crossover and layer crossover, which will result in longitudinal expansions of ropes and bring variations of hoisting velocity and acceleration and, therefore, excite the variation of the hoisting load. Hence, to reveal the change mechanism of hoisting load, the excitations of hoisting velocity and acceleration induced by drum winding should be firstly described.

To analyze for simplicity, a simple LeBus drum with 6 coils shown in Figure 2 was employed to model the winding motion of the rope on the drum. Additionally, the initial winding position of the rope was assumed in the middle of the drum.

As shown in Figure 2, when the rope passes from the initial position 1 to position 2 along the rope groove, it will accomplish the winding of one coil and continually wind into the next coil. During the winding process from the $1^{\text {st }}$ to the $(0.5 N-1)^{\text {th }}$ coil, where $N$ represents the total number of coil per layer, the winding pattern is just coil crossover; at each coil, the rope will wind through two coil crossover 


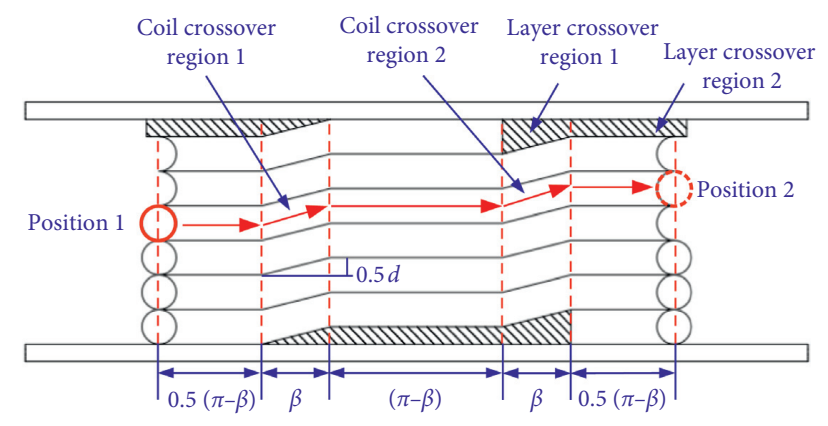

Figure 2: Plane development of rope winding on the drum.

regions as shown in Figure 2. During the winding process at the $(0.5 N)^{\text {th }}$ coil, the layer crossover will occur, making the rope pass from the $1^{\text {st }}$ layer to the $2^{\text {nd }}$ layer. Then, from the $(0.5 N+1)^{\text {th }}$ to the $(1.5 N-1)^{\text {th }}$ coil, the rope will continue to wind on the $2^{\text {nd }}$ layer in the pattern of coil crossover. At the $(1.5 N)^{\text {th }}$ coil, the rope will pass from the $2^{\text {nd }}$ layer to the $3^{\text {rd }}$ layer in the pattern of layer crossover. From the $(1.5 N+1)^{\text {th }}$ coil, the rope will conduct coil crossover on the $3^{\text {rd }}$ layer.

2.1. Excitations from Coil Crossover. During the hoisting process of rope winding on the two symmetrical helical crossover regions, the winding radius will change.

In more detail, during the coil crossover on the first layer, the transverse movement along the main shaft of the drum will accompany the circular winding, which will result in variations of the winding radius. At each coil crossover region on the first layer of the drum as shown in Figure 2, the rope will transversely deflect by $0.5 d \mathrm{~mm}$, where $d$ is the width of the rope groove. That is to say, during the coil crossover region on the first layer, the distance of the rope will wind by $\sqrt{\left(R_{1} \beta\right)^{2}+(0.5 d)^{2}}$ instead of $R_{1} \beta$ where $\beta$ is the corresponding central angle of a single crossover region, and $R_{1}$ is the rated radius of the drum. Therefore, during the rope winding on the coil crossover region on the first layer, the equivalent winding radius will become $r_{1}$ whose equation can be formulated as

$$
r_{1}=\frac{1}{\beta} \sqrt{\left(R_{1} \beta\right)^{2}+\left(\frac{d}{2}\right)^{2}}
$$

During the coil crossover on the second and third layers on the drum, the winding radius will increase. The same as the coil crossover on the first layer, the rope will transversely deflect by $0.5 \mathrm{~d} \cdot \mathrm{mm}$ at each coil crossover region. In addition, the rope will also rise by $h_{1}$ along the radial direction of the drum, where the $h_{1}$ can be expressed as

$$
h_{1}=\left(1-\frac{\sqrt{3}}{2}\right) d \text {. }
$$

According to the spatial winding path of the rope on the second and third layers of the drum as shown in Figure 3, the distance of the rope will wind by $\sqrt{\left(R_{i} \beta\right)^{2}+\left(2 h_{1}\right)^{2}+(0.5 d)^{2}}$ instead of $R_{i} \beta$ where $R_{i}(i=2,3)$ is the equivalent cylindrical radius on the second and third

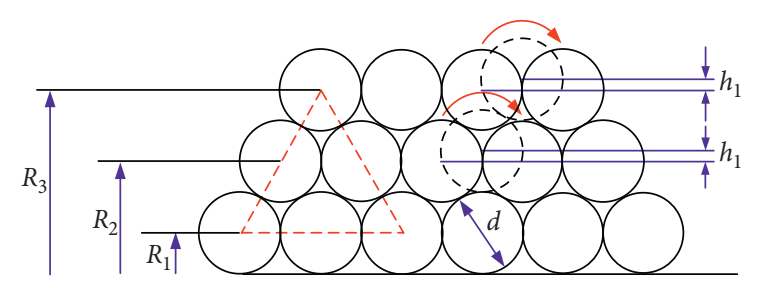

FIgURE 3: Process of coil crossover of rope on the drum.

layers; therefore, the equation of the equivalent winding radius can be calculated as

$$
r_{i}=\frac{1}{\beta} \sqrt{\left(R_{i} \beta\right)^{2}+\left(2 h_{1}\right)^{2}+\left(\frac{d}{2}\right)^{2}}, \quad(i=2,3),
$$

where $r_{i}$ denotes the equivalent winding radius on the coil crossover regions on the $i^{\text {th }}(i=2,3)$ layer.

2.2. Excitations from Layer Crossover. When the rope travels to the $(0.5 N)^{\text {th }}$ coil, it will pass through the layer crossover region 1 into the second layer as shown in Figure 3, realizing the layer crossover and reverse winding. During the winding process at the layer crossover region 1, the rope will climb along the crossover device from the first layer to the second layer by increasing the equivalent radius from $R_{1}$ to $R_{2}$ linearly. In the same manner, when the rope arrives at the $(1.5 N)^{\text {th }}$ coil, it will realize the layer crossover again by increasing the equivalent radius from $R_{2}$ to $R_{3}$, where

$$
R_{i}=R_{1}+(i-1) \frac{\sqrt{3}}{2} d, \quad(i=2,3) .
$$

Therefore, during the first and second layer crossover processes, the distance of the rope will wind by $\sqrt{\left(R_{i} \beta\right)^{2}+(\sqrt{3} / 2 d)^{2}}$ instead of $R_{i} \beta$; therefore, the equivalent winding radiuses $r l_{1}$ and $r l_{2}$ can be formulated by

$$
r l_{i}=\frac{1}{\beta} \sqrt{\left[R_{i} \beta\right]^{2}+\left(\frac{\sqrt{3}}{2} d\right)^{2}}, \quad(i=1,2) .
$$

\subsection{Hoisting Velocity and Acceleration by considering Drum} Excitation. According to the spatial path of the winding rope from initial position 1 as shown in Figure 2 and considering the derived equations (1)-(5), the hoisting velocity, also called winding velocity, can be formulated by

$$
\begin{gathered}
v(t)=w(t) R_{i}, \\
m \in\left[0, \frac{(\pi-\beta)}{2}\right] \cup\left[\frac{(\pi+\beta)}{2}, \frac{(3 \pi-\beta)}{2}\right], \\
v(t)=w(t) R_{i}, \\
m \in\left[\frac{(3 \pi+\beta)}{2}, 2 \pi\right) \text { and } n \neq 0.5 N \text { and } n \neq 1.5 N .
\end{gathered}
$$




$$
\begin{gathered}
v(t)=w(t) R_{i+1}, \\
m \in\left[\frac{(3 \pi+\beta)}{2}, 2 \pi\right) \text { and } n=0.5 N \text { and } n=1.5 N . \\
v(t)=\frac{w(t)}{\beta} \sqrt{\left[R_{i} \beta\right]^{2}+[\delta(i-2)+\delta(i-3)]\left(2 h_{1}\right)^{2}+\left(\frac{d}{2}\right)^{2}}, \\
m \in\left[\frac{(\pi-\beta)}{2}, \frac{(\pi+\beta)}{2}\right] .
\end{gathered}
$$

$$
\begin{gathered}
v(t)=\frac{w(t)}{\beta} \sqrt{\left[R_{i} \beta\right]^{2}+[\delta(i-2)+\delta(i-3)]\left(2 h_{1}\right)^{2}+\left(\frac{d}{2}\right)^{2}}, \\
m \in\left[\frac{(3 \pi-\beta)}{2}, \frac{(3 \pi+\beta)}{2}\right] \text { and } n \neq 0.5 N \text { and } n \neq 1.5 N .
\end{gathered}
$$

$$
\begin{aligned}
& v(t)=\frac{w(t)}{\beta} \sqrt{\left[R_{i} \beta\right]^{2}+\left(\frac{\sqrt{3}}{2} d\right)^{2}}, \\
& m \in\left[\frac{(3 \pi-\beta)}{2}, \frac{(3 \pi+\beta)}{2}\right] \text { and } n=0.5 \mathrm{~N} \text { and } n=1.5 \mathrm{~N},
\end{aligned}
$$

where $t$ is the hoisting time; $w(t)$ is the angular velocity with time; $v(t)$ is the time-varying hoisting velocity; $\delta$ is the Dirac delta function; $m$ is the mathematical operator, which can indicate the region that the rope is passing through according to Figure 2; $n$ represents the number of coils that the rope has winded through. The equations to get variables $m, n$, and $i$ can be formulated as

$$
\begin{aligned}
& n=\operatorname{fix}\left[\frac{\theta(t)}{2 \pi}\right]+1, \\
& m=\operatorname{rem}[\theta(t), 2 \pi], \\
& i= \begin{cases}1, & n \leq 0.5 N, \\
2, & 0.5 N<n \leq 1.5 N, \\
3, & 1.5 N<n,\end{cases}
\end{aligned}
$$

where $\theta(t)$ represents the angle that the drum has rotated by; fix () is the Matlab function that can get the integer part of a decimal number; rem(, ) is the Matlab function that can get the fractional part of a decimal number.

To investigate the change rule of hoisting velocity and acceleration, a three-stage winding scheme of a drum was employed. Taking the motion parameters of the mine hoist given in Table 1 into equations (6)-(14), the hoisting velocity can be calculated as demonstrated in Figure 4. Further, the hoisting acceleration shown in Figure 5 can be obtained by differentiating the calculated hoisting velocity with respect to time.
It can be seen from Figure 4 that the fluctuations of hoisting velocity are induced by the change of the real-time equivalent winding radius during the coil and layer crossover stages. Due to the coil crossover of the rope winding on the drum, the increase of the winding radius leads to the periodic rise of the velocity with amplitude of $0.01 \mathrm{~m} / \mathrm{s}$ as shown in Figure 4(c). What is more, as a result of the layer crossover of the rope, it can be seen from Figure 4(a) that two-step changes appear as shown in regions $\mathrm{A}$ and C. Specifically, from the enlarged views as shown in Figures 4(b) and 4(d), the hoisting velocity will increase by approximately $0.3 \mathrm{~m} / \mathrm{s}$ during the layer crossover processes.

From Figure 5, the velocity variations during the coil and layer crossover stages certainly result in the periodic acceleration impacts. From the enlarged view of region $\mathrm{E}$ shown in Figure 5(c), which corresponds to the stage of coil crossover, periodic alternating acceleration with amplitude of $0.39 \mathrm{~m} / \mathrm{s}^{2}$ will be excited. And during the layer crossover regions shown in Figures 5(b) and 5(d), two transient impact accelerations with amplitudes of $9.64 \mathrm{~m} / \mathrm{s}^{2}$ and $8.35 \mathrm{~m} / \mathrm{s}^{2}$ will be excited.

Hence, it can be concluded that, in a superdeep mine hoisting system powered by LeBus drums, the movement of winding rope will excite the changes of hoisting velocity and acceleration. Specially, the coil crossover of the rope winding on the drum will excite periodic hoisting velocity and acceleration; and the layer crossover will excite step changes of hoisting velocity and acceleration. Additionally, the variations of hoisting velocity and acceleration excited by the layer crossover are much larger than those excited by the coil crossover.

\section{Responses of Dynamic Load}

3.1. Dynamic Model. A model to obtain the dynamic responses is given in Figure 6. Due to the industrial fact that the length of the catenary is much less than the hoisting distance of a superdeep mine hoisting system, the drum and the catenary could be modelled as the vertical configuration. Then, the hoisting rope has a time-varying length $l(t)$ including the catenary length $L$ at time instant $t$, a vertically translating velocity $v(t)=\dot{i}(\mathrm{t})$, and acceleration $a(t)=\ddot{l}(\mathrm{t})$, where the overdot denotes time differentiation. The longitudinal displacements of the rope particle instantaneously located in spatial position $x$ at time $t$ are represented by $u(x, t)$

In the Blair mine hoist, two ropes winded by two drums are applied to hoist the conveyance as shown in Figure 1. The present paper mainly focuses on the effect of drum winding on the dynamic responses of the mine hoisting system; hence, assuming that the two hoisting ropes have the same dynamic properties, and the two drums have the same motion characteristics, only one rope and one drum were adopted to describe the model of hoisting dynamics. In this paper, the eccentricities of the head sheave and the steel guide are not considered. Additionally, due to the constraint of head sheave, the transverse dynamics of the rope can be ignored; only longitudinal vibration of the hoisting rope has been considered to investigate the characteristics of the hoisting system powered by the winding drum. 
TABLE 1: Motion parameters of a superdeep mine hoist.

\begin{tabular}{lcc}
\hline Item & Notation & Value \\
\hline Diameter of hoisting rope & $d$ & $76 \mathrm{~mm}$ \\
Rated radius of drum and head sheave & $R_{1}$ & $4 \mathrm{~m}$ \\
Central angle corresponding to crossover region & $\beta$ & $0.288 \mathrm{rad}$ \\
Number of coils per layer & $\mathrm{N}$ & 30 \\
Winding angular acceleration & $\alpha_{1}$ & $0.1875 \mathrm{rad} / \mathrm{s}^{2}$ \\
Winding angular deceleration & $\alpha_{2}$ & $-0.1875 \mathrm{rad} / \mathrm{s}^{2}$ \\
Uniform winding angular velocity & $w_{0}$ & $4.5 \mathrm{rad} / \mathrm{s}$ \\
Time for winding acceleration & $t_{1}$ & $24 \mathrm{~s}$ \\
Time for uniform winding & $t_{2}$ & $60 \mathrm{~s}$ \\
Time for winding deceleration & $t_{3}$ & $24 \mathrm{~s}$ \\
\hline
\end{tabular}

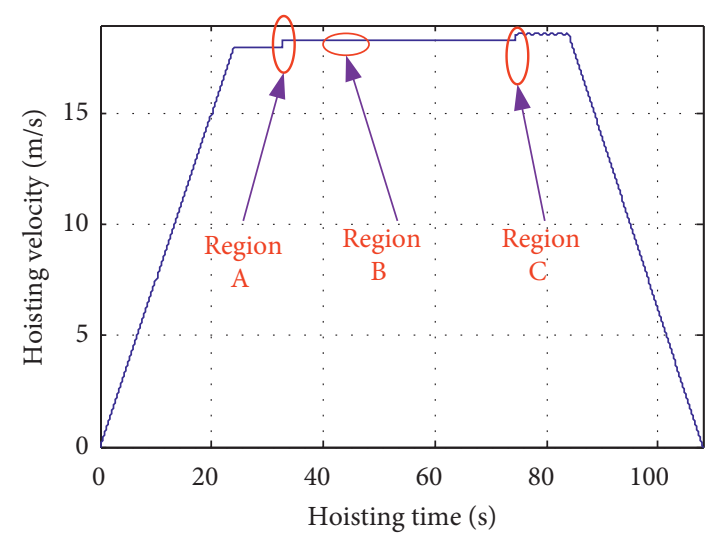

(a)

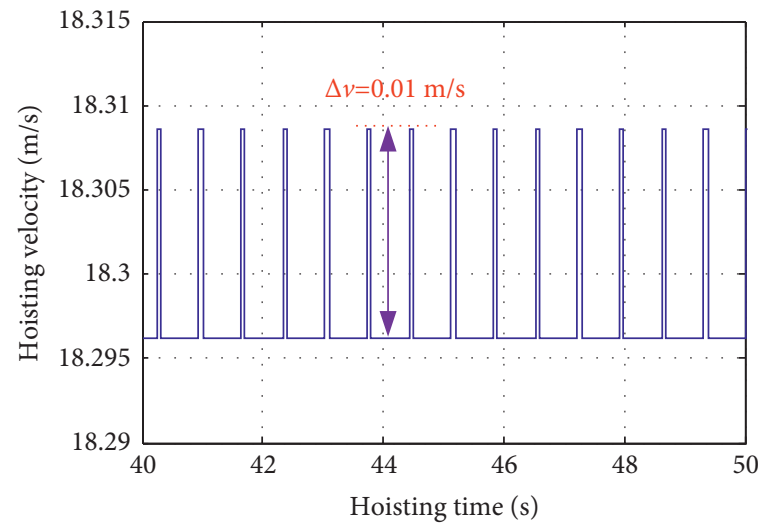

(c)

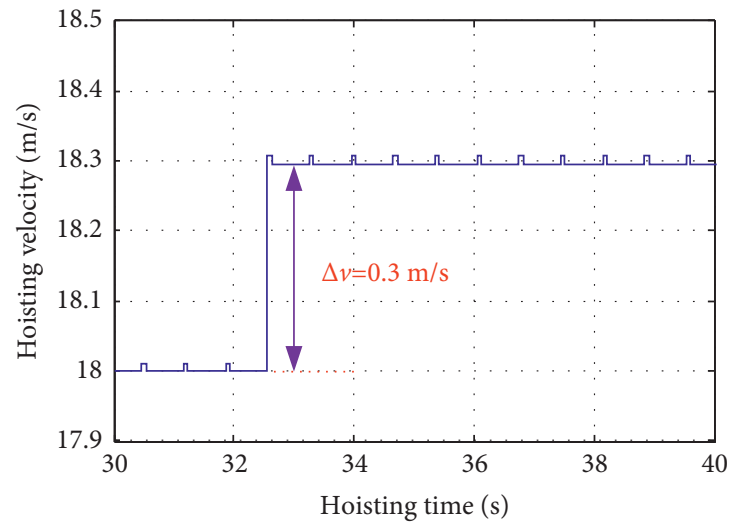

(b)

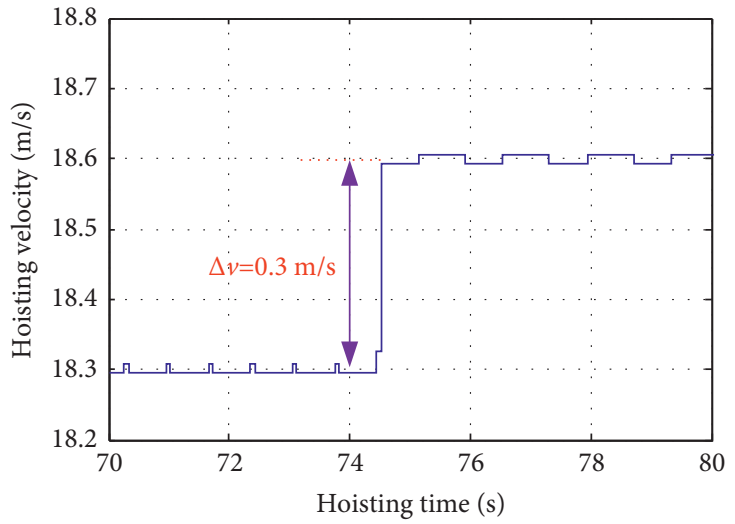

(d)

FIGURE 4: Hoisting velocity derived from rope winding. (a) Hoisting velocity during the whole winding process. (b) Enlarged view of region A. (c) Enlarged view of region B. (d) Enlarged view of region C.

In the present paper, considering the continuity of deflection across the sheave, the longitudinal displacements and strains at both sides of the sheave are considered as equal and continuous. Therefore, the kinetic energy associated with the longitudinal vibration of the rope and conveyance is

$$
\begin{aligned}
E_{k}= & \frac{1}{2} \rho \int_{0}^{l(t)}\left[v(t)+\frac{\mathrm{D} u(x, t)}{\mathrm{D} t}\right]^{2} \mathrm{~d} x+\frac{1}{2}\left(\frac{m_{s}}{2}\right) \\
& {\left[v(t)+\left.\frac{\mathrm{D} u(x, t)}{\mathrm{D} t}\right|_{x=l(t)}\right]^{2}, }
\end{aligned}
$$




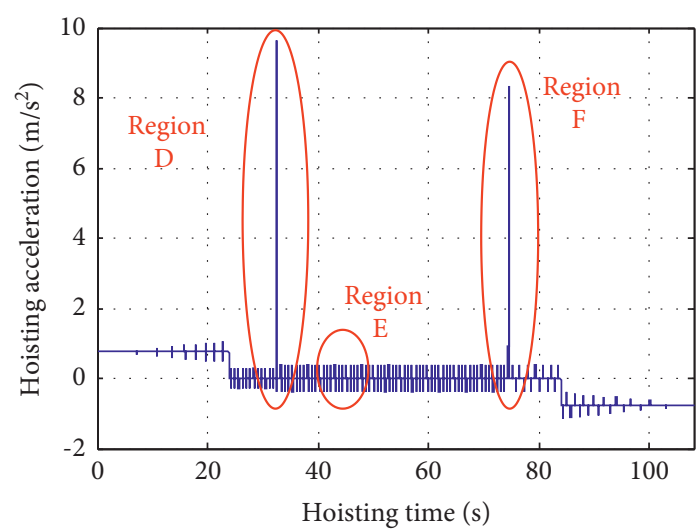

(a)

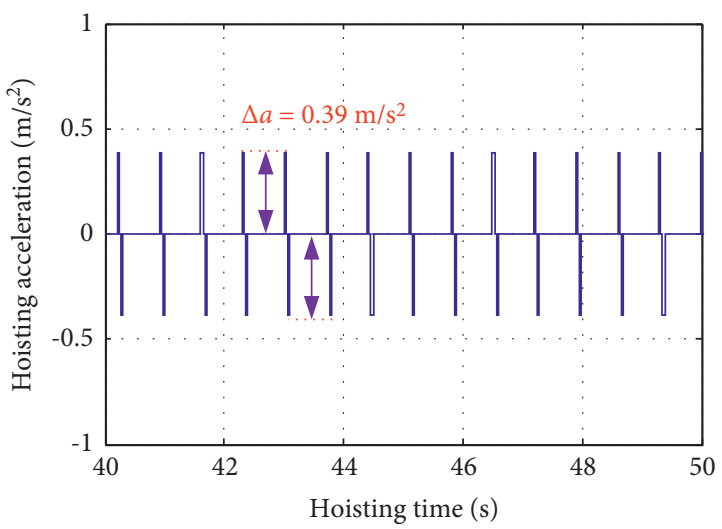

(c)

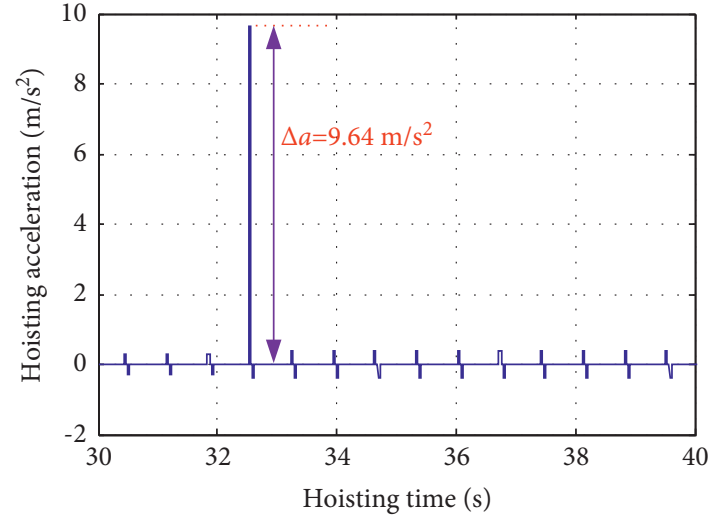

(b)

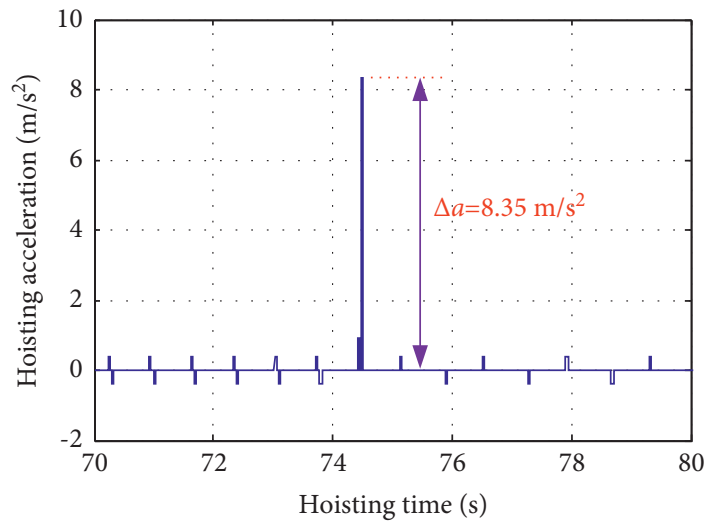

(d)

FIGURE 5: Hoisting velocity derived from rope winding. (a) Hoisting acceleration during the whole winding process. (b) Enlarged view of region D. (c) Enlarged view of region E. (d) Enlarged view of region F.

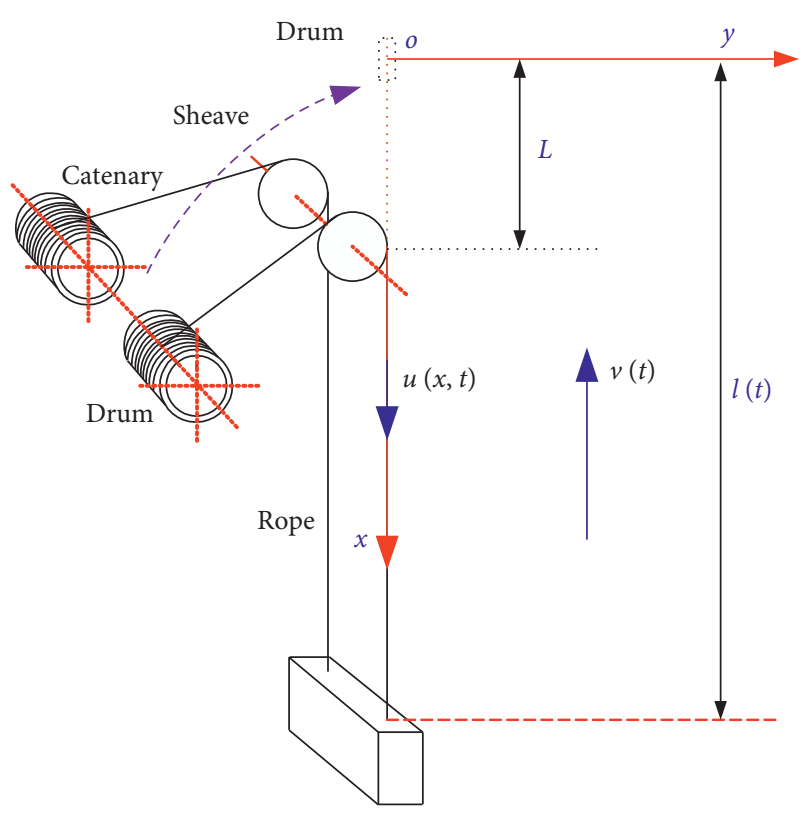

Figure 6: Modeling of longitudinal vibration of hoisting rope.

where $\rho$ is the linear density of the hoisting rope, $m_{\mathrm{s}}$ is the hoisting mass, and $\mathrm{D} / \mathrm{D} t$ is the material derivative, whose equation is

$$
\frac{D}{\mathrm{D} t}=\frac{\partial}{\partial t}+v(x) \frac{\partial}{\partial x}
$$

The potential energy associated with the rope and conveyance is

$$
\begin{aligned}
E_{p}= & \int_{0}^{l(t)} T(x, t) \varepsilon(x, t) \mathrm{d} x+\frac{1}{2} E A \int_{0}^{l(t)} \varepsilon^{2}(x, t) \mathrm{d} x \\
& -\rho g \int_{0}^{l(t)} u(x, t) \mathrm{d} x-\left.\frac{1}{2} m_{\mathrm{s}} g u(x, t)\right|_{x=l(t)},
\end{aligned}
$$

where $T(x, t)$ and $\varepsilon(x, t)$ are the static tension and dynamic strain of the rope instantaneously located in spatial position $x$ at time $t, E A$ is the tensile stiffness of the rope, $g$ is the gravitational acceleration, and the expressions are as follows:

$$
\begin{aligned}
T(x, t) & =\left[\frac{1}{2} m_{s}+\rho(l(t)-x)\right] g, \\
\varepsilon(x, t) & =\frac{\partial u(x, t)}{\partial x}
\end{aligned}
$$

The virtual work done by the damping force is 


$$
\zeta=\int_{0}^{l(t)} C_{u} \frac{\mathrm{D} u(x, t)}{\mathrm{D} t} \delta u(x, t) \mathrm{d} x+\left.C_{\mathrm{s}} \frac{\mathrm{D} u(x, t)}{\mathrm{D} t} \delta u(x, t)\right|_{x=l(t)},
$$

where $C_{\mathrm{u}}$ is the longitudinal damping coefficient of the hoisting rope, and $C_{s}$ is the damping coefficient between the conveyance and the steel guide.

For simplicity, a new independent variable $\xi=x /(t)$ was introduced, and the time-varying spatial domain $[0, l(t)]$ for $x$ can be converted to a fixed domain $[0,1]$ for $\xi$. The new variable is

$$
U(\xi, t)=u(x, t)
$$

The partial derivatives of $(x, t)$ with respect to $t$ and $x$ relative to those of $U(\xi, t)$ with respect to $t$ and $\xi$ are

$$
\begin{aligned}
& \frac{\partial u(x, t)}{\partial t}=\frac{\partial U(\xi, t)}{\partial t}-\xi v(t) l^{-1}(t) \frac{\partial U(\xi, t)}{\partial \xi} . \\
& \frac{\partial u(x, t)}{\partial x}=l^{-1}(t) \frac{\partial U(\xi, t)}{\partial \xi} .
\end{aligned}
$$

Substituting equations (16), (18), (19), and (21)-(23) into equations (15), (17) and (20) yields

$$
\begin{aligned}
E_{k}= & \frac{1}{2} \rho l(t) \int_{0}^{1}\left[v(t)+\frac{\partial U(\xi, t)}{\partial t}+(1-\xi) v(t) l^{-1}(t) \frac{\partial U(\xi, t)}{\partial \xi}\right]^{2} \mathrm{~d} \xi+\frac{m_{s}}{4}[v(t)+\dot{U}(1, t)]^{2}, \\
E_{p}= & \rho g l(t) \int_{0}^{1}(1-\xi) \frac{\partial U(\xi, t)}{\partial \xi} \mathrm{d} \xi+\frac{1}{2} m_{s} g \int_{0}^{1} \frac{\partial U(\xi, t)}{\partial \xi} \mathrm{d} \xi+\frac{1}{2} E A l^{-1}(t) \int_{0}^{1}\left[\frac{\partial U(\xi, t)}{\partial \xi}\right]^{2} \mathrm{~d} \xi \\
& -\rho g l(t) \int_{0}^{1} U(\xi, t) \mathrm{d} \xi-\frac{1}{2} m_{s} g U(1, t), \\
\zeta= & C_{u} l(t) \int_{0}^{1}\left[\frac{\partial U(\xi, t)}{\partial t}+(1-\xi) v(t) l^{-1}(t) \frac{\partial U(\xi, t)}{\partial \xi}\right] \delta U(\xi, t) \mathrm{d} \xi+C_{s} \dot{U}(1, t) \delta U(1, t) .
\end{aligned}
$$

According to the assumed modes method mentioned in the literature [19], the eigenfunctions of a fixed-free string with a unit length are used as the trial functions, and the longitudinal vibration displacement can be approximated by

$$
U(\xi, t)=\sum_{i=1}^{k} \varphi_{i}(\xi) q_{i}(t)
$$

where $k$ is the number of included modes, $q_{i}(t)$, in which $i=1,2, \ldots, k$, are the generalized coordinates, and $\varphi_{i}(\xi)$, in which $i=1,2, \ldots, k$, are the trial functions with the expression as follows:

$$
\varphi_{i}(\xi)=\sin \left(i-\frac{1}{2}\right) \pi \xi, \quad i=1,2, \ldots, k .
$$

It can be noted that, according to the trial functions given in equation (28), the dynamic force of the hoisting rope at the conveyance end is $E A 3(\partial u(l(t), t) / \partial x)=(E A / l(t))$ $3(\partial U(1, t) / \partial \xi)=0$, which cannot satisfy the industrial fact. Therefore, a boundary motion displacement $q_{0}(t)$ was selected to be the spatial derivative of the displacement of the lower end of the rope associated with the trial function $\varphi_{0}(t)$ that meets [19].

$$
\varphi_{0}(\xi)=\xi
$$

Then, the longitudinal vibration displacement of the hoisting rope can be approximated by

$$
U(\xi, t)=\sum_{i=0}^{k} \varphi_{i}(\xi) q_{i}(t)
$$

Lagrange's equations [21] are used as follows:

$$
\begin{aligned}
\frac{\mathrm{d}}{\mathrm{d} t}\left(\frac{\partial E_{k}}{\partial \dot{q}}\right)-\frac{\partial E_{k}}{\partial q}+\frac{\partial E_{p}}{\partial q}+\eta & =0, \\
\eta & =\frac{\partial \zeta}{\partial \delta q},
\end{aligned}
$$

where $\eta$ represents the generalized damping force. Substituting equations (24)-(26), (28)-(30) into equations (31) and (32) yields

$$
\mathrm{M} \ddot{Q}+C \dot{Q}+K Q=F,
$$

where $\mathbf{Q}=\left[q_{0}, q_{1}, q_{2}, \ldots, q_{k}\right]^{T}$ is the vector of generalized coordinates, and $\mathbf{M}, \mathbf{C}, \mathbf{K}$ and $\mathbf{F}$ are the mass, damping, stiffness matrices, and the force vector, respectively, and the expressions are as follows:

$$
\begin{aligned}
M= & \rho l(t) \int_{0}^{1} \phi \phi^{T} \mathrm{~d} \xi+\frac{1}{2} m_{s} \phi(1) \phi^{T}(1) \\
C= & {\left[\rho v(t)+C_{u} l(t)\right] \int_{0}^{1} \phi \phi^{T} \mathrm{~d} \xi+\rho v(t) \int_{0}^{1}(1-\xi) \phi \phi^{\prime T} \mathrm{~d} \xi } \\
& -\rho v(t) \int_{0}^{1}(1-\xi) \phi^{\prime} \phi^{T} \mathrm{~d} \xi+C_{s} \phi(1) \phi^{T}(1),
\end{aligned}
$$




$$
\begin{aligned}
K= & {\left[\rho a(t)+C_{u} v(t)\right] \int_{0}^{1}(1-\xi) \phi \phi^{\prime T} \mathrm{~d} \xi+\rho v^{2}(t) l^{-1}(t) } \\
& \int_{0}^{1} \xi \phi \phi^{\prime T} \mathrm{~d} \xi \\
- & \rho v^{2}(t) l^{-1}(t) \int_{0}^{1}(1-\xi)^{2} \phi^{\prime} \phi^{\prime T} \mathrm{~d} \xi+E A l^{-1}(t) \int_{0}^{1} \phi^{\prime} \phi^{\prime T} \mathrm{~d} \xi \\
F= & {\left[\rho g l(t)-\rho a(t) l(t)-\rho v^{2}(t)\right] \int_{0}^{1} \phi \mathrm{d} \xi-\left[\rho g l(t)-\rho v^{2}(t)\right] } \\
& \int_{0}^{1}(1-\xi) \phi \prime \mathrm{d} \xi \\
- & \frac{1}{2} m_{s} g \int_{0}^{1} \phi \prime \mathrm{d} \xi+\frac{1}{2} m_{s}[g-a(t)] \phi(1) .
\end{aligned}
$$

where $\varphi=\left[\varphi_{0}, \varphi_{1}, \varphi_{2}, \ldots, \varphi_{k}\right]^{T}$, and the superscript (') denotes partial differentiation with respect to $\xi$.

3.2. Impact Responses of Hoisting Load. By applying Runge-Kutta method to solve equations (33)-(37) using the hoisting velocity and acceleration given in Figures 4(a) and 5(a), and the hoisting parameters presented in Table 2, the dynamic displacement and force at the conveyance end of the rope were calculated and are demonstrated in Figures 7 and 8 .

Figure 7 shows the variation trend of dynamic displacement at the conveyance end of the hoisting rope. At the start and end points of the acceleration and deceleration periods such as the points $\mathrm{G}, \mathrm{H}$, and $\mathrm{K}$, abrupt oscillations of the displacement will appear and last for a period of time. Besides, displacement oscillations will also be excited by the impact accelerations caused by coil and layer crossover of the winding rope on the LeBus drum. Furthermore, seen from points I, J in Figure 7, the vibration displacements caused by layer crossover are much more than those caused by coil crossover. Therefore, it can be concluded that the layer crossover of rope winding on LeBus drum has a greater influence on the dynamic displacement of the hoisting conveyance than coil crossover.

Figure 8(a) demonstrates dynamic force at the conveyance end of the hoisting rope, and it can be seen that the vibratory load will be excited by the impact acceleration caused by coil and layer crossover. Furthermore, by subtracting static load from the dynamic force in Figure 8(a), the impact load induced by the impact acceleration was obtained as shown in Figure 8(b). It can be seen that two peak values of $64430 \mathrm{~N}$ and $76380 \mathrm{~N}$ of impact loads caused by layer crossover will be excited, and the vibratory impact loads will continue for a period of time.

Though the vibratory displacements caused by coil crossover are so small, and they almost approach zero as shown in Figure 7, the vibratory loads caused by coil crossover cannot be neglected, and from Figure 8(c), alternating impact load with maximum peak value of $3387 \mathrm{~N}$ and maximum valley value of $1635 \mathrm{~N}$ will be excited. Additionally, during the deceleration period, the alternating impact load will continue with peak-to-peak value of approximately $44580 \mathrm{~N}$.

From all the analysis above, it can be concluded firstly that the coil and layer crossover of the rope winding on the LeBus drum will excite impact displacement and load of the hoisting rope; subsequently, coil and layer crossover have a greater influence on the impact load than displacement; and the layer crossover has a greater influence on the impact displacement and load than coil crossover. Eventually, coil and layer crossover of rope winding on the drum will excite alternating impact displacement and load of the hoisting rope, which will result in fatigue damage of the rope and accelerate the rupture of the rope, seriously decreasing the reliability of the machine and the safety of coal production.

\section{Experimental Validation}

To verify the validity of the established model and impact responses of the system, a test rig was built to carry out the measurement. As demonstrated in Figure 9, the test rig was constructed according to winding-type mine hoist, and a steel wire rope was designed to pass from the cylindrical drum over a head sheave to the conveyance constrained to move in the vertical direction. The cylindrical drum was connected with a retarder by a flexible coupling. And a servo motor, which was directly connected to the retarder, was used to power the test rig. The hoisting velocity and acceleration could be set by the control program on the computer, which can send valid instructions to the motor driver.

To follow the property of coil and layer crossover of the rope winding on the drum, as shown in Figure 10, two cylinder objects were symmetrically tied onto the surface of the cylindrical drum. For simplicity in modeling, the initial position of the rope was assumed as shown in Figure 10. Due to the existence of the two cylinder objects on the surface of the cylindrical drum, two winding transition zones with the corresponding central angle $\beta_{1}$ were thereby induced during the winding process. And according to the geometric relationship between the cylindrical drum and the wrapped objects, during the two transition zones, an additional winding length of $\delta \mathrm{l}=5.1835 \mathrm{~mm}$ would be induced. Furthermore, the equivalent winding radius $\delta R$ can be formulated by

$$
\delta R=\frac{R_{t} \beta_{1}+\delta l}{\beta_{1}}
$$

where $R_{t}$ is the radius of the cylindrical drum. Therefore, the actual winding velocity $v(t)$ and acceleration $a(t)$ of the test rig can be calculated as 
TABLE 2: Hoisting parameters of a superdeep mine hoist.

\begin{tabular}{lcc}
\hline Item & Notation & Value \\
\hline Linear density of the rope & $\mathrm{P}$ & $21.4 \mathrm{~kg} / \mathrm{m}$ \\
Tensile stiffness of the rope & $E A$ & $m_{\mathrm{s}}$ \\
Hoisting mass & $C_{\mathrm{u}}$ & $6.67310^{8} \mathrm{~N} / \mathrm{m}$ \\
Longitudinal damping coefficient of the hoisting rope & $C_{\mathrm{s}}$ & $50000 \mathrm{~kg}$ \\
Damping coefficient between the conveyance and the steel guide & $L$ & 100 \\
Length of the catenary & $l_{\min }$ & $85 \mathrm{~m}$ \\
Minimum length of the vertical rope & $20 \mathrm{~m}$ \\
\hline
\end{tabular}

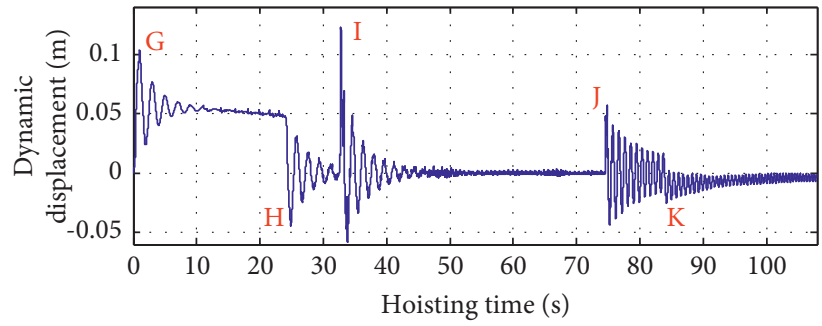

FIGURE 7: Modeling of longitudinal vibration of hoisting rope.

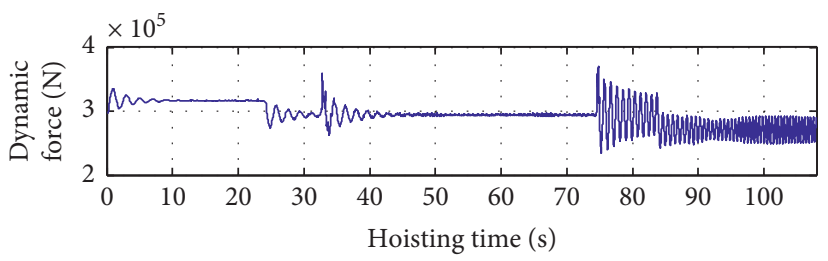

(a)

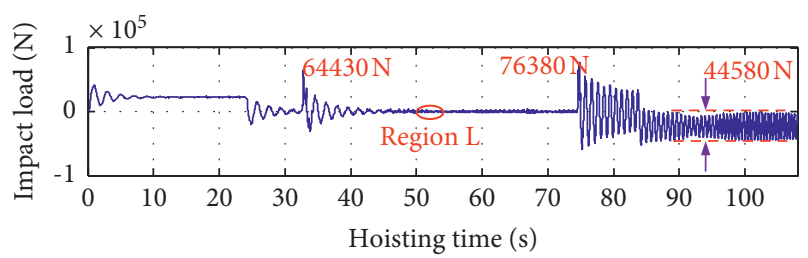

(b)

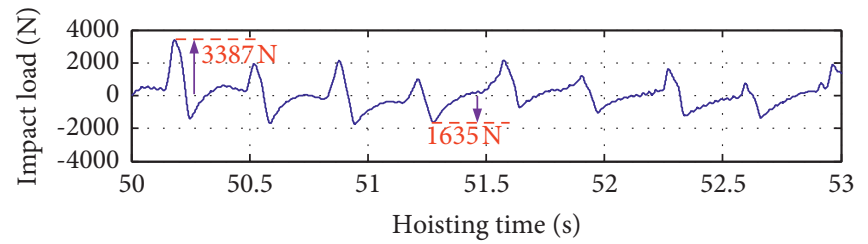

(c)

Figure 8: Dynamic load at the conveyance end of the rope. (a) Axial dynamic force at the conveyance end of the rope. (b) Impact load at the conveyance end of the rope. (c) Enlarged view of the region L.
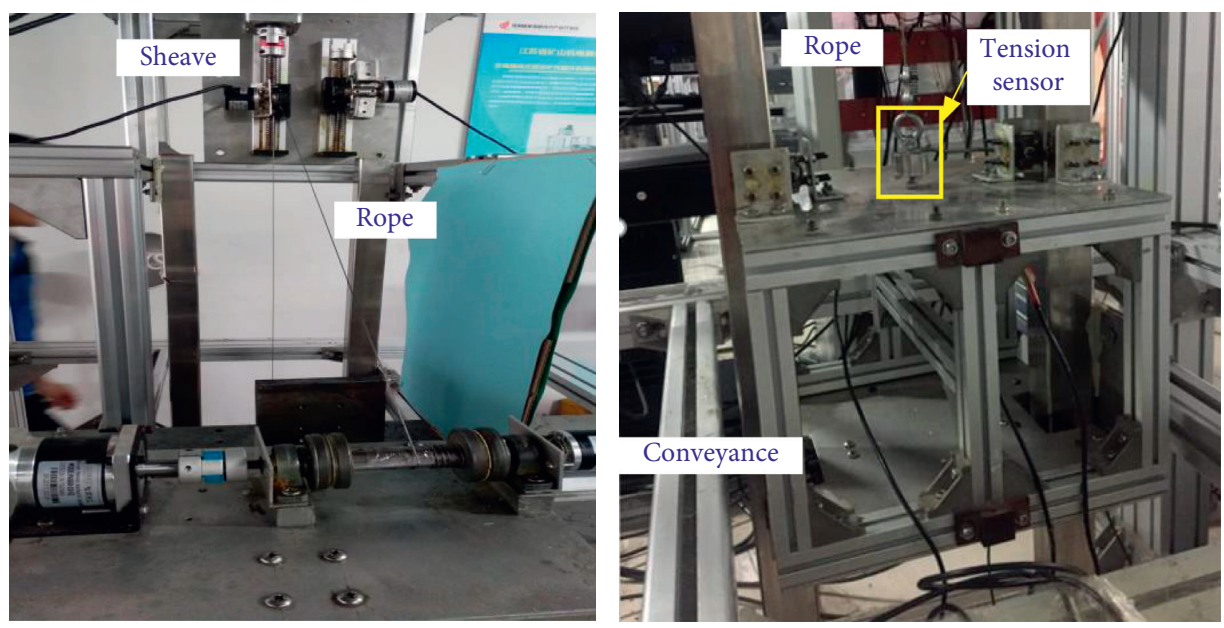

Figure 9: Test rig. 

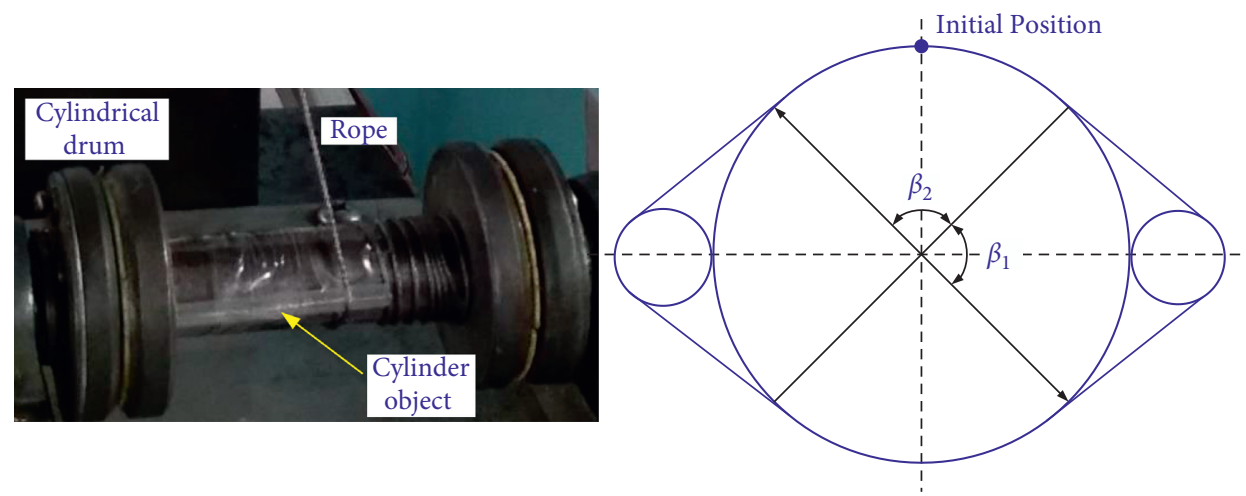

FIgURE 10: Excitation setting.

Table 3: Parameters of test rig.

\begin{tabular}{lcc}
\hline Item & Notation & \multicolumn{1}{c}{ Value } \\
\hline Winding angular acceleration & $\alpha_{1 \mathrm{t}}$ & $50 \mathrm{rad} / \mathrm{s}^{2}$ \\
Winding angular deceleration & $\alpha_{2 \mathrm{t}}$ & $-50 \mathrm{rad} / \mathrm{s}^{2}$ \\
Time for winding acceleration & $t_{1 \mathrm{t}}$ & $0.2 \mathrm{~s}$ \\
Time for uniform winding & $t_{2 \mathrm{t}}$ & $6.4 \mathrm{~s}$ \\
Time for winding deceleration & $t_{3 \mathrm{t}}$ & $0.2 \mathrm{~s}$ \\
Linear density of the rope & $\rho_{\mathrm{t}}$ & $0.4 \mathrm{~kg} / \mathrm{m}$ \\
Tensile stiffness of the rope & $E A_{\mathrm{t}}$ & $1288.1 \mathrm{~N} / \mathrm{m}$ \\
Hoisting mass & $m_{\mathrm{st}}$ & $1.49 \mathrm{~kg}$ \\
Radius of drum and sheave & $R_{\mathrm{t}}$ & $0.01 \mathrm{~m}$ \\
Diameter of cylinder object & $d_{\mathrm{t}}$ & $0.0045 \mathrm{~m}$ \\
Longitudinal damping coefficient of the hoisting rope & $C_{\mathrm{ut}}$ & 1.5 \\
Damping coefficient between the conveyance and the steel guide & $C_{\mathrm{st}}$ & 0.1 \\
Length of the catenary & $L_{\mathrm{t}}$ \\
Minimum length of the vertical rope & $l_{\mathrm{tmin}}$ & $1 \mathrm{~m}$ \\
\hline
\end{tabular}

$$
\begin{aligned}
& \left\{\begin{array}{l}
v_{t}(t)=w_{t}(t) R_{t}, m_{t} \in\left[0, \frac{\beta_{2}}{2}\right] \cup\left[\frac{\beta_{2}}{2}+\beta_{1}, \frac{3 \beta_{2}}{2}+\beta_{1}\right] \cup\left[\frac{3 \beta_{2}}{2}+2 \beta_{1}, 2 \pi\right] \\
a_{t}(t)=\alpha_{t}(t) R_{t},
\end{array}\right. \\
& \left\{\begin{array}{l}
v_{t}(t)=w_{t}(t) \delta R, \\
a_{t}(t)=\alpha_{t}(t)\left(R_{t}+\frac{\delta l}{\beta_{1}}\right), \\
m_{t} \in\left[\frac{\beta_{2}}{2}, \frac{\beta_{1}+\beta_{2}}{2}\right] \cup\left[\frac{3 \beta_{2}}{2}+\beta_{1}, \frac{3}{2}\left(\beta_{1}+\beta_{2}\right)\right], \\
a_{t}(t)=\alpha_{t}(t)\left(R_{t}-\frac{\delta l}{\beta_{1}}\right),
\end{array}\right. \\
& \left\{\begin{array}{l}
v_{t}(t)=w_{t}(t) \delta R, \\
m_{t}=\operatorname{rem}\left[\theta_{t}(t), 2 \pi\right]
\end{array}\right.
\end{aligned}
$$

where $\theta_{t}(t)$ represents the angle that the drum has rotated by; $\operatorname{rem}($,$) is the Matlab function that can get the fractional part$ of a decimal number.

A tension sensor was installed between the rope and conveyance in order to obtain the dynamic force. And the tension data were recorded by data acquisition card and sent to the computer.
Applying the geometric and motion parameters of the test rig given in Table 3, the hoisting velocity and acceleration of the test rig have been calculated by using equations (38)-(42) and shown in Figure 11. By applying Runge-Kutta method to solve equations (33)-(37) using the hoisting velocity and acceleration given in Figure 11 and the hoisting parameters presented in Table 3, the dynamic force at the 


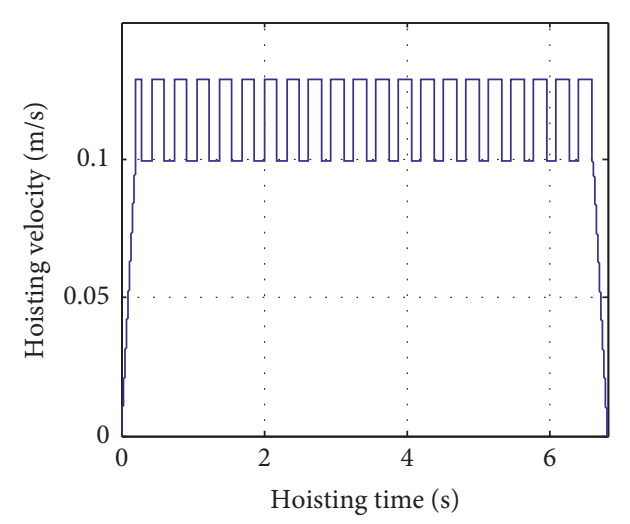

(a)

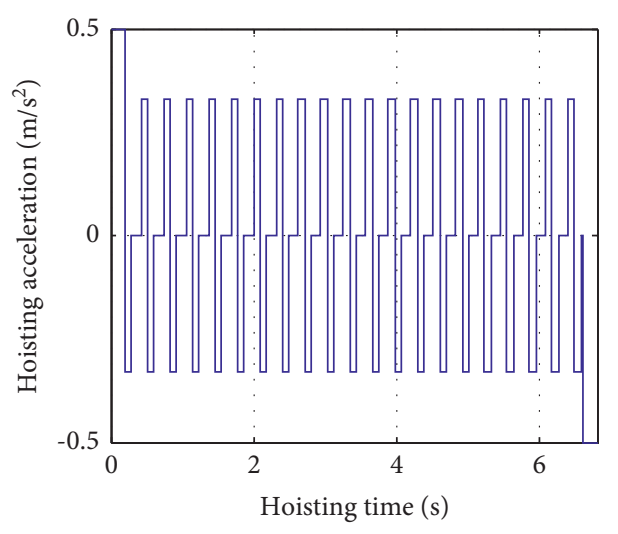

(b)

FIGURE 11: Hoisting profile of the test rig. (a) Hoisting velocity of the test rig. (b) Hoisting acceleration of the test rig.

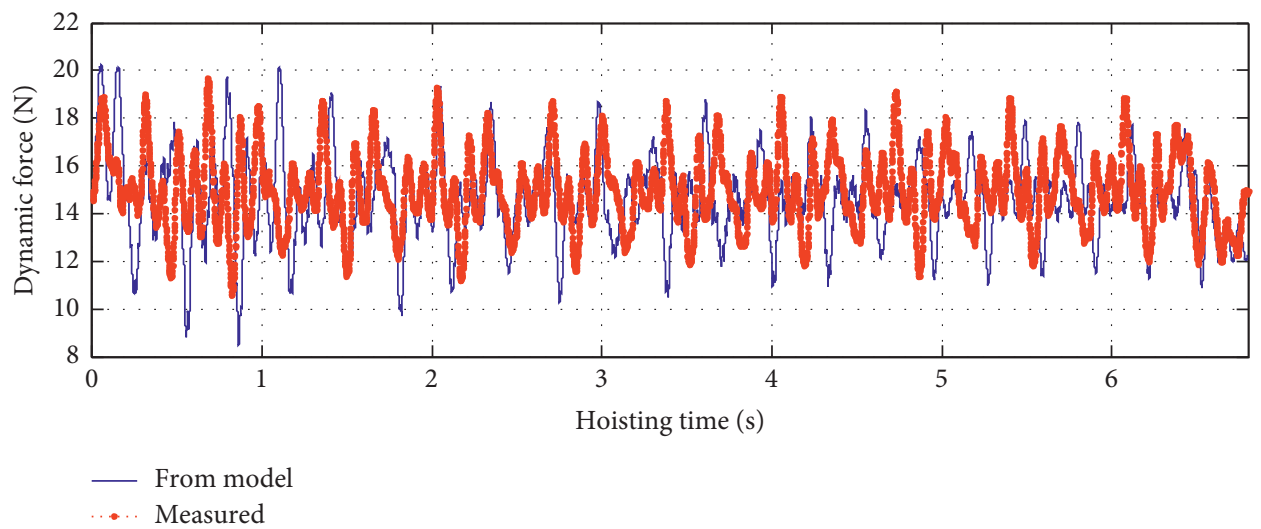

FIGURE 12: Dynamic forces at the conveyance end of the rope from model calculation and measurement.

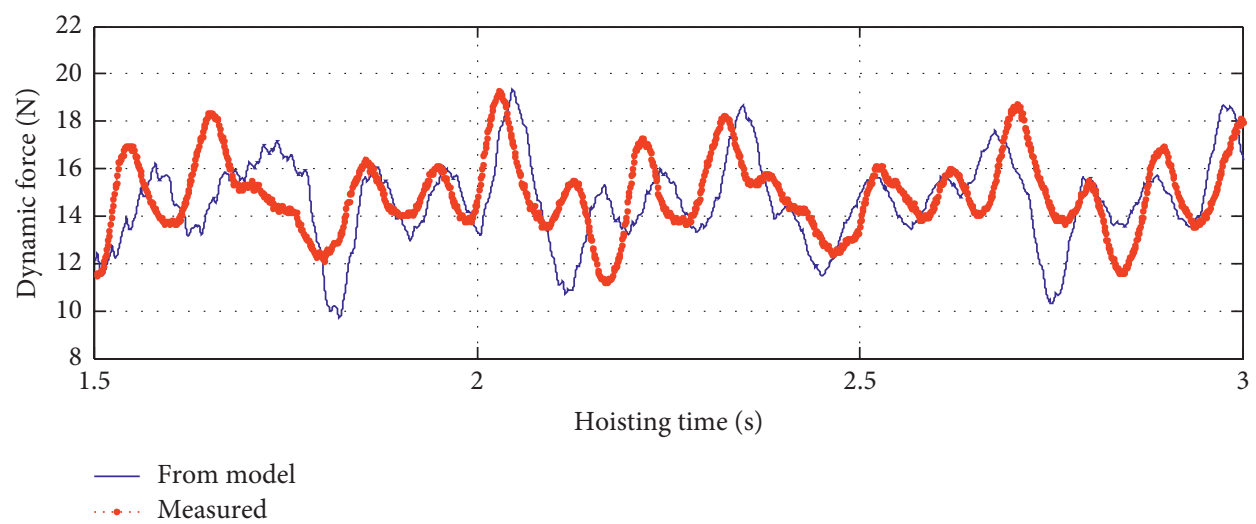

Figure 13: Enlarged view of the dynamic forces at the conveyance end of the rope.

conveyance end of the rope was calculated and is demonstrated in Figure 12 as well as the measured force from the tension sensor.

It can be seen from Figures 12 and 13 that the vibratory trend and numerical value of the dynamic force at the conveyance end of the rope from the model calculation are well consistent with those from the measured. Additionally, it should be noted that stochastic excitations and nonlinear disturbances of the test rig are difficult to be modelled and are not the main influence factors; therefore, the stochastic excitations and nonlinear disturbances of the test rig were not considered during the modeling process, and it is 
reasonable that some minor differences of the numerical values between the force from the model and measured exist.

What is more, it can be seen from Figure 13 that the good consistent trend can verify the validity of the established model, and the investigated effect of drum winding on the dynamic responses of the hoisting rope analyzed in Section 3 has also been validated. Therefore, compared to the existing studies demonstrated in the introductory part, the highlight of the present paper is that the impact mechanism of the drum winding such as the coil crossover and layer crossover on the load response in the superdeep mine hoisting systems has been explored theoretically and validated experimentally.

\section{Conclusions}

The effect of drum winding on impact load in superdeep mine hoisting systems has been investigated in detail. Firstly, the hoisting velocity and acceleration with step changes have been precisely modelled and calculated, indicating that the variations of hoisting velocity and acceleration excited by the layer crossover are much larger than those excited by the coil crossover. Secondly, a dynamic model, which can calculate the dynamic displacement and force of the rope, was established, and the impact responses of the system were obtained by the established model, indicating that coil and layer crossover have a greater influence on the impact load than displacement; and the layer crossover has a greater influence on the impact responses than coil crossover. The paper will provide a good theoretical foundation for designing and optimizing the mine hoists in superdeep mine shafts in order to realize stable operation of the hoisting system.

\section{Data Availability}

The data used to support the findings of this study are available from the corresponding author upon request.

\section{Conflicts of Interest}

The authors declare that there are no conflicts of interest regarding the publication of this article.

\section{Acknowledgments}

The authors gratefully acknowledge the support of National Natural Science Foundation of China (Grant nos. 51805273 and 51975569) and the support of Qing Lan Project of Jiangsu Province and the Priority Academic Program Development of Jiangsu Higher Education Institutions (PAPD).

\section{References}

[1] X. Peng, X. S. Gong, and J. J. Liu, "Vibration control on multilayer cable moving through the crossover zones on mine hoist," Shock and Vibration, vol. 2016, Article ID 6878021, 7 pages, 2016.
[2] X. Peng, X.-S. Gong, and J.-J. Liu, "The study on crossover layouts of multi-layer winding grooves in deep mine hoists based on transverse vibration characteristics of catenary rope," Proceedings of the Institution of Mechanical Engineers Part I: Journal of Systems \& Control Engineering, vol. 233, no. 2, pp. 118-132, 2018.

[3] J. Yao, Y. Ma, C. Ma, T. Xu, and X. Xiao, "Prediction and reduction of spatial transverse vibration of hoisting catenaries induced by drum winding in super-deep mine hoists," International Journal of Modelling, Identification and Control, vol. 36, no. 2, pp. 159-173, 2020.

[4] J. Wang, Y. Pi, Y. Hu, and X. Gong, "Modeling and dynamic behavior analysis of a coupled multi-cable double drum winding hoister with flexible guides," Mechanism and Machine Theory, vol. 108, pp. 191-208, 2017.

[5] S. Kaczmarczyk and W. Ostachowicz, "Transient vibration phenomena in deep mine hoisting cables. part 1: mathematical model," Journal of Sound and Vibration, vol. 262, no. 2, pp. 219-244, 2003.

[6] S. Kaczmarczyk and W. Ostachowicz, "Transient vibration phenomena in deep mine hoisting cables. part 2: numerical simulation of the dynamic response," Journal of Sound and Vibration, vol. 262, no. 2, pp. 245-289, 2003.

[7] Y. D. Wang, G. H. Cao, Z. C. Zhu, J. J. Wang, and N. G. Wang, "Longitudinal response of parallel hoisting system with timevarying rope length," Journal of Vibroengineering, vol. 16, no. 8, pp. 1392-8716, 2014.

[8] Y. B. Guo, D. K. Zhang, X. Y. Zhang, S. Q. Wang, W. Ma, and M. Terzo, "Experimental study on the nonlinear dynamic characteristics of wire rope under periodic excitation in a friction hoist," Journal of Shock and Vibration, vol. 2020, Article ID 8506016, 14 pages, 2020.

[9] Y. Guo, D. Zhang, K. Chen, C. Feng, and S. Ge, "Longitudinal dynamic characteristics of steel wire rope in a friction hoisting system and its coupling effect with friction transmission," Tribology International, vol. 119, pp. 731-743, 2018.

[10] J. H. Huang, C. X. Luo, P. Yu, and H. M. Hao, "A methodology for calculating limit deceleration of flexible hoisting system: a case study of mine hoist," Journal of Proceedings of the Institution of Mechanical Engineers, Part E: Journal of Process Mechanical Engineering, vol. 234, no. 4, pp. 1-11, 2020.

[11] J. Wu and Z. Kou, "Theoretical coupling longitudinaltransverse model and experimental verification of transverse vibration of rope for multi-rope friction hoisting system," Journal of International Journal of Coal Science \& Technology, vol. 3, no. 1, pp. 77-84, 2016.

[12] G. H. Cao, J. J. Wang, and Z. C. Zhu, "Coupled vibrations of rope-guided hoisting system with tension difference between two guiding ropes," Proc IMechE Part C: Journal of Mechanical Engineering Science, vol. 232, no. 2, pp. 231-244, 2018.

[13] Q. F. Peng, P. Xu, H. Yuan et al., "Analysis of vibration monitoring data of flexible suspension lifting structure based on time-varying theory," Journal of Sensors, vol. 20, no. 22, pp. 1-19, 2020.

[14] Q. Zhang, Y. H. Yang, T. Hou, and R. J. Zhang, "Dynamic analysis of high-speed traction elevator and traction car-rope time-varying system," Journal of Noise \& Vibration Worldwide, vol. 50, no. 2, pp. 37-45, 2019.

[15] Q. Zhang, T. Hou, R. J. Zhang, and J. Liu, "Time-varying characteristics of the longitudinal vibration of a high-speed traction elevator lifting system," International Journal of Acoustics and Vibration, vol. 25, no. 2, pp. 153-161, 2020. 
[16] N. G. Wang, G. H. Cao, L. Yan, and L. Wang, "Modelling and passive control of flexible guiding hoisting system with timevarying length," Journal of Mathematical and Computer Modelling of Dynamical Systems, vol. 26, no. 1, pp. 31-54, 2020.

[17] D. H. Yang, K. Y. Kim, M. K. Kwak, and S. J. Lee, "Dynamic modeling and experiments on the coupled vibrations of building and elevator ropes," Journal of Sound and Vibration, vol. 390, pp. 164-191, 2017.

[18] X. Arrasate, S. Kaczmarczyk, G. Almandoz, J. M. Abete, and I. Isasa, "The modeling, simulation and experimental testing of the dynamic responses of an elevator system," Mechanical Systems and Signal Processing, vol. 42, pp. 258-282, 2014.

[19] W. D. Zhu and H. Ren, "An accurate spatial discretization and substructure method with application to moving elevator cable-car systems-part I: methodology," Journal of Vibration and Acoustics, vol. 135, pp. 1-10, Article ID 051036, 2013.

[20] H. Ren and W. D. Zhu, "An accurate spatial discretization and substructure method with application to moving elevator cable-car systems-part II: application," Journal of Vibration and Acoustics, vol. 135, pp. 1-21, Article ID 051037, 2013.

[21] R. M. Rosenberg, Analytical Dynamics of Discrete Systems, Plenum Press, New York, NY, USA, 1977. 